\title{
The Ocular Surface Characteristics in Prostate Cancer Patients Treated with Androgen Deprivation Therapy
}

\author{
Weina Li, ${ }^{1,2,3}$ Xiaofeng Li, ${ }^{1}$ Feilun Cui, ${ }^{4}$ Zhipeng Xu, ${ }^{4}$ Nuo Dong $\mathbb{D}^{1,2}$ and Cheng $L i \mathbb{D}^{1,2}$ \\ ${ }^{1}$ Eye Institute \& Affiliated Xiamen Eye Center, School of Medicine, Xiamen University, Xiamen, China \\ ${ }^{2}$ Fujian Provincial Key Laboratory of Ophthalmology and Visual Science \& Ocular Surface and Corneal Diseases, Xiamen, China \\ ${ }^{3}$ Department of Ophthalmology, Changhai Hospital, Shanghai, China \\ ${ }^{4}$ Department of Urology, Zhenjiang First People's Hospital, Zhenjiang, China
}

Correspondence should be addressed to Nuo Dong; profeye@163.com and Cheng Li; cheng-li@xmu.edu.cn

Weina Li and Xiaofeng Li contributed equally to this work.

Received 8 September 2021; Accepted 22 October 2021; Published 9 November 2021

Academic Editor: Wen-Qing Shi

Copyright (c) 2021 Weina Li et al. This is an open access article distributed under the Creative Commons Attribution License, which permits unrestricted use, distribution, and reproduction in any medium, provided the original work is properly cited.

Background.To investigate the association of long-term androgen deprivation therapy (ADT) with ocular surface characteristics in prostate cancer patients. Methods. A total of 30 male prostate cancer patients who received ADT were selected. All candidates were scored using the Ocular Surface Disease Index (OSDI) and subsequently divided into two groups containing 9 symptomatic patients (scores $>12$ ) and 21 asymptomatic patients (scores $\leq 12$ ). Another 20 healthy age-matched males were selected as the control group. Each candidate was assessed with respect to eyelid margin abnormality, tear film break-up time (NI-BUT), tear meniscus height (TMH), meiboscore, meibum expressibility, and demodex infection. Results. The NI-BUT in the ADT group was significantly shorter than that in the control group. The scores for OSDI, eyelid margin abnormality, meibum expressibility, and meiboscores were significantly higher in the ADT group $(P<0.05)$. Moreover, the NI-BUT in the symptomatic ADT group was significantly shorter than that in the asymptomatic ADT group $(P<0.05)$. The meiboscores and meibum expressibility score in the symptomatic ADT group were significantly higher than those in the asymptomatic ADT group $(P<0.05)$. The presence of demodex in the symptomatic ADT group was also higher than that in the asymptomatic ADT group $(P<0.05)$. The length of time that patients had been taking ADT was positively correlated with meiboscores and negatively correlated with NI-BUT. Conclusion. Androgen levels were associated with significant changes in relative meibomian gland function. Subjective symptoms, such as dryness and foreign body sensation, were more obvious in prostate cancer patients receiving ADT, which may be caused by MGD and demodex infection. It's recommended that more attention be paid to the ocular surface in prostate cancer patients taking ADT by performing examination of NI-BUT and meibomian gland morphology and function with a view to providing more comprehensive prevention and treatment protocols.

\section{Introduction}

Prostate cancer is the most common malignant tumor in men, and its incidence is gradually increasing annually. As early as 1941, Huggins and Hodges proposed androgen deprivation as an effective treatment for prostate cancer [1]. Nowadays, androgen deprivation therapy (ADT) is the mainstay of treatment for locally recurrent or advanced prostate cancer [2]. Although ADT is customized based on individual disease characteristics, side effects of these drugs are inevitable due to the long-term lack of androgen regulation. The effect of meibomian gland damage on the development of dry eye after long-term ADT has rarely been evaluated.

The meibomian glands can synthesize and secrete lipids to form the superficial tear film layer. Meibomian gland dysfunction (MGD) is a chronic diffuse abnormality of the meibomian glands, characterized by terminal duct obstruction and/or qualitative/quantitative changes in glandular secretion [3]. MGD is thought to be caused by abnormal lipid excretion that results in increased evaporation, leading to 

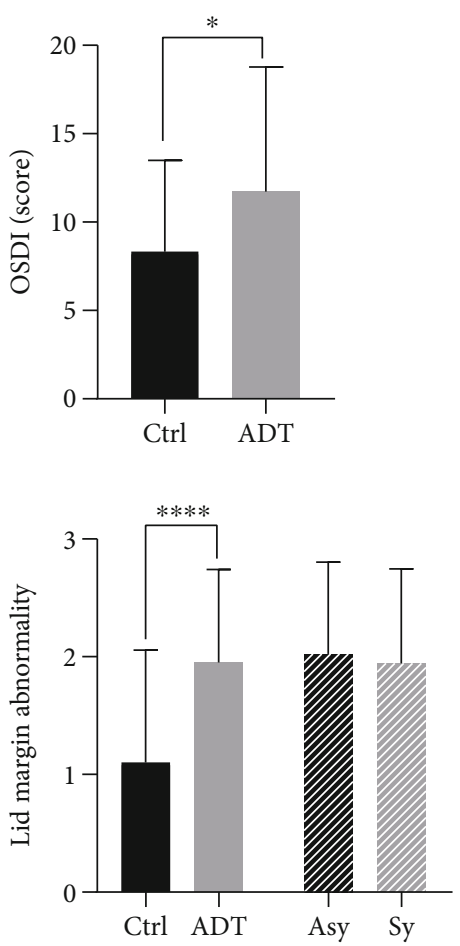
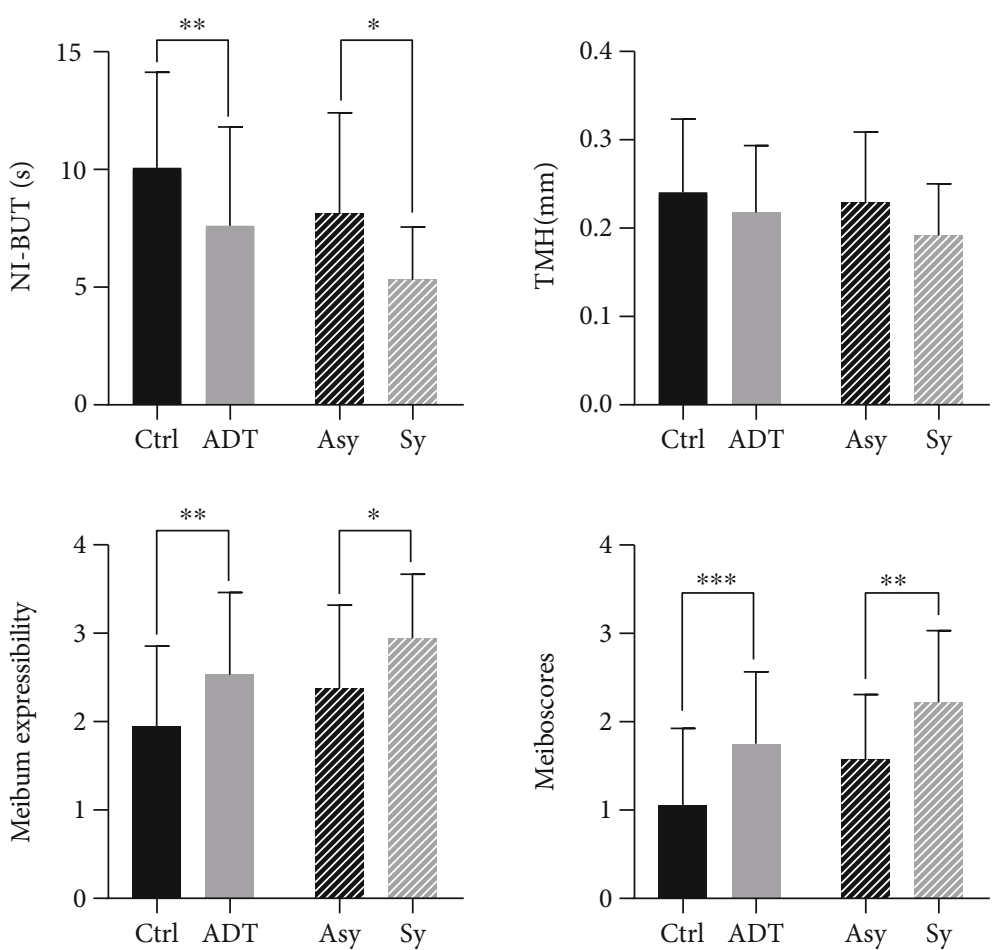

FIGURE 1: Mann-Whitney $U$ test of each examined item between prostate cancer patients receiving ADT and the control group, as well as between the asymptomatic and symptomatic ADT groups $\left({ }^{* * *} P<0.001,{ }^{* *} P<0.01,{ }^{*} P<0.05\right)$.

evaporative dry eye disease (DED). Previous studies have shown that androgens can exert a protective function on the ocular surface [4].

The meibomian glands are an androgen target. They express androgen receptor proteins in acinar epithelial cell nuclei [5], in addition to mRNAs for type 1 and/or type 2 $5 \alpha$-reductase, which convert testosterone to the very potent dihydrotestosterone [6].

Androgens play a key role in meibomian gland physiology, stimulating function and suppressing keratinization [7]. Schirra et al. [8] demonstrated that androgens regulate the expression of more than 1590 genes involved mainly in lipid metabolism in the mouse meibomian gland. Moreover, androgen deficiency is a risk factor for MGD and evaporative DED [7, 9]. MGD and DED have been reported to be associated with age and gender, the prevalence of which is higher in the elderly and in women $[7,10]$. Although there is some evidence supporting the ability of chronic androgen deficiency to alter lipid secretion in meibomian glands, leading to MGD and DED [11], there is no clinical confirmation of their relationship with symptoms of dry eye or treatment time in patients taking androgen deprivation drugs.

To date, there have been only limited investigations regarding the possible association between androgens and meibomian glands. Given this background, we predict that androgen deficiency will influence meibomian gland function, alter meibum secretion, and decrease tear film stability, and that abnormality of the ocular surface in prostate cancer patients is related to the length of time taking ADT. To test this hypothesis, we sought to determine the effects of androgen deficiency on the ocular surface and meibomian glands by comparing prostate cancer patients receiving long-term $\mathrm{ADT}$ with age-matched male controls.

\section{Materials and Methods}

The study sample comprised 30 male prostate cancer patients $(74.77 \pm 8.62$ years old, $n=60)$ receiving longterm ADT and 20 age-matched male controls $(72.30 \pm 6.19$ years old, $n=40$ ) who had not received ADT. The average duration of ADT in prostate cancer patients was 15.06 months (range 3-60 months). For the ADT group, prostate cancer patients receiving ADT was the sole inclusion criterion. The exclusion criteria were infectious keratitis or conjunctivitis, continuous eye drop use, history of eye surgery, and serious systematic disease. All participants were recruited from Zhenjiang First People's Hospital between June 2018 and December 2019.

2.1. Symptom Assessment. Dry eye symptoms were assessed using the OSDI Questionnaire, which comprises 12 questions regarding the frequency of ocular discomfort, visual problems interfering with daily activity, and ocular discomfort caused by environmental factors [12]. Total OSDI was calculated using the following formula: OSDI $=[$ (sum of all answered questions $) \times 100] /$ [total number of answered questions) $\times 4$ ]. Total scores were expressed on a scale of 0 to 100 , with higher scores representing a greater disability [13]. Subjects with a score $>12$ were placed into the symptomatic group, and the rest were placed into the asymptomatic group. 
TABLE 1: Ocular surface assessment and statistical results among the groups. Data are shown as the mean \pm SD. $P$ value was calculated using the Mann-Whitney $U$ test.

\begin{tabular}{|c|c|c|c|c|c|c|}
\hline & Ctrl & $\mathrm{ADT}$ & $P$ & Asymptomatic ADT group & Symptomatic ADT group & $P$ \\
\hline Age & $72.30 \pm 6.19$ & $74.77 \pm 8.62$ & 0.276 & $72.22 \pm 7.88$ & $75.61 \pm 8.97$ & 0.418 \\
\hline OSDI & $8.31 \pm 1.15$ & $11.72 \pm 1.28$ & 0.04 & - & - & - \\
\hline NI-BUT (s) & $10.07 \pm 4.05$ & $7.60 \pm 4.20$ & 0.006 & $8.13 \pm 4.27$ & $5.32 \pm 2.23$ & 0.033 \\
\hline $\mathrm{TMH}(\mathrm{mm})$ & $0.24 \pm 0.08$ & $0.21 \pm 0.07$ & 0.134 & $0.22 \pm 0.07$ & $0.19 \pm 0.05$ & 0.085 \\
\hline Lid margin abnormality score & $1.10 \pm 0.95$ & $1.95 \pm 0.79$ & $\leq 0.001$ & $2.02 \pm 0.78$ & $1.94 \pm 0.80$ & 0.666 \\
\hline Meibum expressibility & $1.95 \pm 0.90$ & $2.53 \pm 0.92$ & 0.007 & $2.38 \pm 0.93$ & $2.94 \pm 0.72$ & 0.013 \\
\hline Meiboscores & $1.05 \pm 0.87$ & $1.75 \pm 0.81$ & $\leq 0.001$ & $1.57 \pm 0.73$ & $2.22 \pm 0.80$ & 0.005 \\
\hline
\end{tabular}

TABLE 2: Demodex infection rate among the groups.

\begin{tabular}{|c|c|c|c|c|}
\hline & Ctrl & $\mathrm{ADT}$ & Symptomatic ADT group & Asymptomatic ADT group \\
\hline Demodex infection rate (\%) & $60.00 \%$ & $70.00 \%$ & $100 \%$ & $57.14 \%$ \\
\hline$X^{2}$ & \multicolumn{2}{|c|}{0.53} & \multicolumn{2}{|c|}{-} \\
\hline$P$ & \multicolumn{2}{|c|}{$0.46^{\mathrm{a}}$} & \multicolumn{2}{|c|}{$0.029^{\mathrm{b}}$} \\
\hline
\end{tabular}

2.2. Noninvasive Tear Film Break-Up Time. All subjects underwent imaging with a Keratograph (Oculus, Wetzlar, Germany) that measured the noninvasive tear film breakup time (NI-BUT). Subjects focused on the central target, and a Placido disc pattern comprising 22 illuminated rings was projected onto the corneal surface. Subjects were asked to blink twice and keeped their eyes open until the next eye blink or the sampling procedure ended. Then, the first NIBUT was recorded.

2.3. Tear Meniscus Height. The tear meniscus height (TMH) was evaluated using a Keratograph that had four infrared diodes under deactivation of red ring illumination and measured perpendicular to the eyelid margin at the 6 o'clock position of the corneal midline [14]. All examinations were performed three times.

2.4. Meibographic Evaluation. Meibography was accomplished using the infrared camera system of the oculus Keratograph. The meibomian glands were visualized by everting the eyelid. Areas of meibomian gland dropout and total tarsal plate were measured to calculate the percentage of meibomian gland dropout. Meiboscores were assessed according to the grading scale as described by Arita et al. [15]: grade 0 , no loss of meibomian glands; grade 1, area loss $<33 \%$ of the total meibomian gland area; grade 2 , area loss between $33 \%$ and $67 \%$ of the total meibomian gland area; and grade 3 , area loss $>67 \%$ of the total meibomian gland area.

2.5. Eyelid Margin Abnormality. Under a slit lamp microscope, eyelid margin abnormality was quantitated as the total score of the following 4 parameters: plugged meibomian gland orifices, eyelid margin irregularity, eyelid margin vascular engorgement, and mucocutaneous junction displacement. The score ranged from 0 to 4 depending on the number of abnormalities present [16].
2.6. Examination of Demodex Infection. A total of 4 eyelashes from each eyelid were removed using fine forceps and placed on a glass slide. A drop of $70 \%$ glycerinum was added to the lashes before a coverslip was mounted. Under a light microscope, demodex was identified based on morphology and movement.

2.7. Meiboscores. After placing a hot compress on the eyelid for 10 minutes, pressure was applied to the upper and lower eyelid using two cotton buds to encourage the meibomian glands to secrete lipids. Characteristics of the meibum were graded on a numerical scale of $0-3$ as follows [17]: grade 0 , clear fluid; grade 1, cloudy fluid; grade 2, cloudy fluid with granular debris; and grade 3, thick toothpaste-like fluid. The higher the meiboscore, the cloudier and more viscous the meibum.

2.8. Statistical Analysis. The data were analyzed using SPSS 26.0 (Inc, Chicago, Illinois, USA) and are expressed as the mean \pm standard deviation. Comparison of clinical parameters between the ADT and control groups was performed using the Mann-Whitney $U$ test, $X^{2}$ test, and Fisher's exact probability test. Correlations between clinical parameters in the ADT group were evaluated by Spearman's correlation. $P$ $<0.05$ was considered statistically significant.

\section{Results}

A total of 100 eyes (ADT group, 60; control group, 40) were evaluated. Subjects in both groups were age-matched elderly males. The reference value for serum testosterone in healthy men was $1.75-7.81 \mathrm{ng} / \mathrm{mg}$. This study selected prostate cancer patients whose serum testosterone value was $0.10 \pm 0.13 \mathrm{ng} /$ $\mathrm{mg}$, which is significantly below normal.

The dry eye symptoms in prostate cancer patients receiving long-term ADT were more severe than those in control individuals (Figure 1). In comparison with the control group, the ADT group exhibited a significantly shorter NI-BUT $(P=0.004$; Table 1$)$. The OSDI, eyelid margin abnormality 
TABle 3: Correlation between the duration of ADT and each ocular test. $P$ value was calculated using Spearman's correlation test. $P<0.05$ was considered statistically significant.

\begin{tabular}{|c|c|c|c|c|c|c|}
\hline & NI-BUT (s) & $\begin{array}{l}\mathrm{TMH} \\
(\mathrm{mm})\end{array}$ & $\begin{array}{l}\text { Lid margin abnormality } \\
\text { score }\end{array}$ & $\begin{array}{c}\text { Meibum } \\
\text { expressibility }\end{array}$ & Meiboscores & $\begin{array}{c}\text { Time and } \\
\text { ADT }\end{array}$ \\
\hline NI-BUT (s) & RA & & & & & \\
\hline \multirow{2}{*}{$\mathrm{TMH}(\mathrm{mm})$} & $R=-0.144$ & \multirow{2}{*}{ RA } & & & & \\
\hline & $P=0.274$ & & & & & \\
\hline \multirow{2}{*}{$\begin{array}{l}\text { Lid margin abnormality } \\
\text { score }\end{array}$} & $R=0.076$ & $R=-0.048$ & \multirow{2}{*}{ RA } & & & \\
\hline & $P=0.565$ & $P=0.713$ & & & & \\
\hline \multirow{2}{*}{ Meibum expressibility } & $R=0.02$ & $R=0.067$ & $R=0.157$ & \multirow{2}{*}{ RA } & & \\
\hline & $P=0.882$ & $P=0.611$ & $P=0.23$ & & & \\
\hline \multirow{2}{*}{ Meiboscores } & $R=-0.116$ & $R=0.144$ & $R=-0.016$ & $R=0.390^{* *}$ & \multirow{2}{*}{ RA } & \\
\hline & $P=0.377$ & $P=0.272$ & $P=0.901$ & $P=0.002$ & & \\
\hline \multirow{2}{*}{ Time and ADT } & $R=-0.563^{* *}$ & $R=0.161$ & $R=-0.006$ & $R=0.304^{*}$ & $R=0.132$ & \multirow{2}{*}{ RA } \\
\hline & $P \leq 0.001$ & $P=0.22$ & $P=0.963$ & $P=0.018$ & $P=0.316$ & \\
\hline
\end{tabular}

score, and mean meiboscores as well as meibum expressibility scores in the ADT group were significantly higher than those in the control group (all, $P<0.05$; Table 1); however, there was no significant difference in TMH between the two groups.

According to the OSDI scores, prostate cancer patients were divided into the symptomatic ADT group (scores $>12$ ) containing 9 patients and the asymptomatic ADT group (scores $\leq 12$ ) containing 21 patients. Following subgroup analysis of patients with established dry eye symptoms and those without, we found that the NI-BUT in the symptomatic ADT group was significantly shorter than that in the asymptomatic ADT group $(P<0.05)$. The mean meiboscores and meibum expressibility scores in the symptomatic ADT group were significantly higher than those in the asymptomatic ADT group $(P<0.05)$. Ocular surface discomfort was associated with a decrease in NI-BUT. There were no significant differences in the eyelid margin abnormality score or $\mathrm{TMH}$ between the symptomatic and asymptomatic ADT groups according to the completion of a questionnaire designed to assess dry eye symptoms.

Previous research has shown that demodex infestation is closely related to meibomian gland dysfunction and ocular surface inflammation [18]. In the present research, the percentage of demodex infection was $60.0 \%$ and $70.0 \%$ in the $\mathrm{ADT}$ and control group, respectively. There was no significant difference in demodex infection rate between these two groups $(P>0.05$; Table 2$)$. However, following grouping of the prostate cancer patients based on OSDI score, all 9 symptomatic patients had demodex infection. For the symptomatic and asymptomatic groups, the demodex infection rate was $100 \%$ and $57.14 \%$, respectively. The difference was statistically significant $(P<0.05$; Table 2$)$.

MGD is a common and chronic progressive disease that has an adverse impact on the ocular surface. The duration of ADT as a time-dependent variable was examined for its association with MGD. In the present study, correlation analysis between clinical parameters in the ADT group (Table 3) revealed that the treatment time was positively correlated with the meiboscores and negatively correlated with the NI-BUT.

\section{Discussion}

Androgens are known to induce the proliferation and differentiation of sebaceous glands throughout the body [19]. Given that the meibomian glands are large sebaceous glands that express androgen receptors, androgens also play an important role in meibomian gland development. However, long-term ADT suppresses androgen levels, which can slow or stop the growth of prostate cancer, but this leads to physiological changes in the ocular surface. The present study investigated the changes in the structure and function of the ocular surface in prostate cancer patients taking ADT. These patients experienced a greater frequency of tear film debris, irregular eyelid margins, atrophy and orifice metaplasia of the meibomian glands, and a marked decrease in the quality of meibomian gland secretions than age-matched controls.

Dry eye syndrome is a relatively common. Analysis of the OSDI scores results indicated that ADT group did have a higher frequency of dry eye syndrome. Tear film stability is evaluated by measuring the NI-BUT. There was a significant decrease in NI-BUT values in the ADT group as compared with those in the control group, demonstrating a decrease in tear film stability. The eyelid margin abnormality, meibum expressibility, and meiboscores were higher than those in the control group, indicating changes in the morphology and function of meibomian glands in patients taking ADT. Androgens have been shown to significantly affect lipid metabolic pathways [20], and the synthesis and/or elaboration of lipids by the meibomian glands is thought to be regulated by androgens [5]. A previous study showed that ADT can exert a significant and consistent influence on the mass/charge ratios of neutral lipid fraction of meibomian gland secretions [21]. Moreover, Jennifer et al. [22] reported that androgen insensitivity can cause 
meibomian gland dysfunction and significantly increase dry eye signs and symptoms in patients with complete androgen insensitivity syndrome (CAIS). Androgens can also modulate the morphology, physiology, molecular biology, immunology, and secretion of the lacrimal gland [23]. Interestingly, Sullivan et al. [24] demonstrated that ADT has no influence on the level of tear secretion in humans. Similarly, no significant difference in $\mathrm{TMH}$ between the two groups was found in the present study. Androgen deficiency appears to cause the development of meibomian gland dysfunction and evaporative dry eye.

Prostate cancer can be aggressive and metastasize to the bones and regional lymph nodes. There are no reports of metastasis to the eye; therefore, prostate cancer itself does not cause tear function-related damage to the ocular surface. To further investigate the reasons for dry eye in prostate cancer patients treated with ADT, we divided these patients into symptomatic and asymptomatic subgroups based on the OSDI score. Our results indicate that the NI-BUT was shorter in the symptomatic ADT group than that in the asymptomatic ADT group. A significant difference was noted in meibum expressibility scores and meiboscores between these two groups $(P<0.05$ for each), indicating that the tear film stability was worse in the symptomatic ADT group. Meibomian glands secrete lipids into the tear film, forming a superficial lipid layer that can maintain tear film stability. It can be concluded that the reason for tear film instability in the ADT group, especially in those with dry eye symptoms, may be abnormalities in the meibomian glands.

Demodex mites are a potential cause of MGD [25]. The demodex infestation rate was significantly higher in the symptomatic ADT group $(9 / 9,100.00 \%$, Table 2$)$ than that in the asymptomatic ADT group, indicating that the ocular discomfort experienced by prostate cancer patients was related to ocular demodex infestation. Franz et al. [26] reported that the prevalence of demodex in patients with ocular discomfort was high. Moreover, Lee et al. [27] showed that the number of demodex mites was proportional to the OSDI score and the severity of ocular discomfort. Taken together, this evidence implies that demodex infestation can cause an increase in the subjective symptoms of ocular surface damage. Thus, eradication of demodex may be beneficial in relieving ocular surface discomfort related to MGD.

MGD has many underlying causative factors such as age, gender, and hormones. Aging may impact atrophy of meibomian gland acini epithelial cells and reduce the quality of meibomian gland secretions [28]. Decreased sex hormone levels, especially androgens, which are correlated with advancing age, may contribute to meibomian gland loss [24], leading to increased viscosity of the meibum, hyperkeratinization of the meibomian gland orifices, and meibomian gland obstruction [29]. MGD due to androgen deficiency has been demonstrated in prostate cancer patients receiving ADT [11]; however, no studies to date have reported an association of ADT duration with changes in the ocular surface. In the present study, the duration of ADT was positively associated with the meibomian gland dropout score in the ADT group but negatively correlated with the NIBUT. This indicates that a longer duration of ADT was associated with poorer tear function and a larger meibomian gland dropout area. Similarly, decreased sebaceous gland function and secretion are correlated with both an atrophy of acinar cells and a reduction in serum androgen levels [30]. Meibomian gland atrophy tends to increase with age, and a significant correlation between age and loss of meibomian gland area was observed by infrared meibography in a previous study [14]. In short, the androgen level is associated with meibomian gland alterations. Thus, we speculated that androgens may stimulate meibomian gland function, enhancing tear film stability and decreasing tear film evaporation and consequently ameliorating dry eye symptoms. A longer duration of ADT can lead to a significant increase in the risk of MGD.

To the best of our knowledge, this is the first study to investigate the association between long-term ADT and the risk of MGD in an Asian population. Our study focused on the relationship between androgens and the ocular surface and found that androgen deficiency was related to ocular discomfort, eyelid margin abnormality, and meibomian gland loss. In conclusion, we demonstrate that long-term ADT in prostate cancer patients increases the risk of MGD. Moreover, demodex infection can aggravate ocular surface discomfort. Monitoring ocular surface health and treating demodex are crucial in prostate cancer patients taking ADT.

\section{Data Availability}

All data used to support the findings of this study is available on request.

\section{Conflicts of Interest}

The authors declare that there is no potential conflict of interest relevant to the publication of this article.

\section{Authors' Contributions}

Weina Li and Xiaofeng Li contributed equally to this study.

\section{Acknowledgments}

This study was supported in part by grants from The National Key R\&D Program of China (2020YFA0908100), the National Natural Science Foundation of China (NSFC Nos. 81970771, 82070931, 81770891, 81672955), the Health Research Talent Training Program of Fujian PRC (2016-ZQN-90), Xiamen Key Medical and Health Project (No. 3502Z20191101), Zhenjiang Science Technology Planning Project (No. SH2019033), and the Huaxia Translational Medicine Fund for Young Scholars (Nos. 2017-A-001, 2017-A-002).

\section{References}

[1] C. Huggins and C. V. Hodges, "Studies on prostatic cancer. I. The effect of castration, of estrogen and androgen injection on serum phosphatases in metastatic carcinoma of the prostate," CA: a Cancer Journal for Clinicians, vol. 22, no. 4, pp. 232-240, 1972.

[2] E. M. Bolton and T. Lynch, "Are all gonadotrophin-releasing hormone agonists equivalent for the treatment of prostate 
cancer? A systematic review," BJU International, vol. 122, no. 3, pp. 371-383, 2018.

[3] J. D. Nelson, J. Shimazaki, J. M. Benitez-del-Castillo et al., "The international workshop on meibomian gland dysfunction: report of the definition and classification subcommittee," Investigative Opthalmology \& Visual Science, vol. 52, no. 4, pp. 1930-1937, 2011.

[4] D. A. Sullivan, B. D. Sullivan, J. E. Evans et al., "Androgen deficiency, meibomian gland dysfunction, and evaporative dry eye," Annals of the New York Academy of Sciences, vol. 966, no. 1, pp. 211-222, 2002.

[5] D. A. Sullivan, B. D. Sullivan, M. D. Ullman et al., "Androgen influence on the meibomian gland," Investigative Ophthalmology \& Visual Science, vol. 41, no. 12, pp. 3732-3742, 2000.

[6] E. M. Rocha, L. A. Wickham, L. A. da Silveira et al., "Identification of androgen receptor protein and 5alpha-reductase mRNA in human ocular tissues," British Journal of Ophthalmology, vol. 84, no. 1, pp. 76-84, 2000.

[7] D. A. Schaumberg, J. J. Nichols, E. B. Papas, L. Tong, M. Uchino, and K. K. Nichols, "The international workshop on meibomian gland dysfunction: report of the subcommittee on the epidemiology of, and associated risk factors for, MGD," Investigative Ophthalmology \& Visual Science, vol. 52, no. 4, pp. 1994-2005, 2011.

[8] F. Schirra, T. Suzuki, S. M. Richards et al., "Androgen control of gene expression in the mouse meibomian gland," Investigative Ophthalmology \& Visual Science, vol. 46, no. 10, pp. 3666-3675, 2005.

[9] D. A. Sullivan, A. Bélanger, J. M. Cermak et al., "Are women with Sjögren's syndrome androgen-deficient?," The Journal of Rheumatology, vol. 30, no. 11, pp. 2413-2419, 2003.

[10] J. A. Smith, J. Albeitz, C. Begley et al., "The Epidemiology of Dry Eye Disease: Report of the Epidemiology Subcommittee of the International Dry Eye WorkShop (2007)," The Ocular Surface, vol. 5, no. 2, pp. 93-107, 2007.

[11] K. L. Krenzer, M. R. Dana, M. D. Ullman et al., "Effect of androgen deficiency on the human meibomian gland and ocular surface," The Journal of Clinical Endocrinology and Metabolism, vol. 85, no. 12, pp. 4874-4882, 2000.

[12] B. E. Dougherty, J. J. Nichols, and K. K. Nichols, "Rasch analysis of the Ocular Surface Disease Index (OSDI)," Investigative Ophthalmology \& Visual Science, vol. 52, no. 12, pp. 8630-8635, 2011.

[13] R. M. Schiffman, M. D. Christianson, G. Jacobsen, J. D. Hirsch, and B. L. Reis, "Reliability and validity of the Ocular Surface Disease Index," Archives of Ophthalmology, vol. 118, no. 5, pp. 615-621, 2000.

[14] R. Arita, K. Itoh, K. Inoue, and S. Amano, "Noncontact infrared meibography to document age-related changes of the meibomian glands in a normal population," Ophthalmology, vol. 115, no. 5, pp. 911-915, 2008.

[15] R. Arita, K. Itoh, S. Maeda et al., "Proposed diagnostic criteria for obstructive meibomian gland dysfunction," Ophthalmology, vol. 116, no. 11, pp. 2058-2063.e1, 2009.

[16] H. Lee, K. Min, E. K. Kim, and T. I. Kim, "Minocycline controls clinical outcomes and inflammatory cytokines in moderate and severe meibomian gland dysfunction," American Journal of Ophthalmology, vol. 154, no. 6, pp. 949-957.e1, 2012.

[17] A. J. Bron, L. Benjamin, and G. R. Snibson, "Meibomian gland disease. Classification and grading of lid changes," Eye, vol. 5, no. 4, pp. 395-411, 1991.
[18] X. Luo, J. Li, C. Chen, S. Tseng, and L. Liang, "Ocular demodicosis as a potential cause of ocular surface inflammation," Cornea, vol. 36, no. 1, pp. S9-S14, 2017.

[19] A. Azmahani, Y. Nakamura, K. M. McNamara, and H. Sasano, "The role of androgen under normal and pathological conditions in sebaceous glands: the possibility of target therapy," Current Molecular Pharmacology, vol. 9, no. 4, pp. 311-319, 2016.

[20] D. M. Kelly and T. H. Jones, "Testosterone: a metabolic hormone in health and disease," The Journal of Endocrinology, vol. 217, no. 3, pp. R25-R45, 2013.

[21] B. D. Sullivan, J. E. Evans, K. L. Krenzer, M. Reza Dana, and D. A. Sullivan, "Impact of antiandrogen treatment on the fatty acid profile of neutral lipids in human meibomian gland secretions," The Journal of Clinical Endocrinology and Metabolism, vol. 85, no. 12, pp. 4866-4873, 2000.

[22] J. M. Cermak, K. L. Krenzer, R. M. Sullivan, M. R. Dana, and D. A. Sullivan, "Is complete androgen insensitivity syndrome associated with alterations in the meibomian gland and ocular surface?," Cornea, vol. 22, no. 6, pp. 516-521, 2003.

[23] H. Wagner, B. A. Fink, and K. Zadnik, "Sex- and gender-based differences in healthy and diseased eyes," Optometry, vol. 79, no. 11, pp. 636-652, 2008.

[24] D. A. Sullivan, K. L. Krenzer, B. D. Sullivan, D. B. Tolls, I. Toda, and M. R. Dana, "Does androgen insufficiency cause lacrimal gland inflammation and aqueous tear deficiency?," Investigative Ophthalmology \& Visual Science, vol. 40, no. 6, pp. 1261-1265, 1999.

[25] Y. Y. Gao, M. A. di Pascuale, A. Elizondo, and et al.S. C. G. Tseng, "Clinical Treatment of Ocular Demodecosis by Lid Scrub With Tea Tree Oil," Cornea, vol. 26, no. 2, pp. 136143, 2007.

[26] D. F. Rabensteiner, H. Aminfar, I. Boldin et al., "Demodex mite infestation and its associations with tear film and ocular surface parameters in patients with ocular discomfort," American Journal of Ophthalmology, vol. 204, pp. 7-12, 2019.

[27] S. H. Lee, Y. S. Chun, J. H. Kim, E. S. Kim, and J. C. Kim, "The relationship betweenDemodexand ocular discomfort," Investigative Ophthalmology \& Visual Science, vol. 51, no. 6, pp. 2906-2911, 2010.

[28] B. D. Sullivan, J. E. Evans, M. R. Dana, and D. A. Sullivan, "Influence of aging on the polar and neutral lipid profiles in human meibomian gland secretions," Archives of Ophthalmology, vol. 124, no. 9, pp. 1286-1292, 2006.

[29] E. Knop, N. Knop, T. Millar, H. Obata, and D. A. Sullivan, “The international workshop on meibomian gland dysfunction: report of the subcommittee on anatomy, physiology, and pathophysiology of the meibomian gland," Investigative Ophthalmology \& Visual Science, vol. 52, no. 4, pp. 1938-1978, 2011.

[30] J. L. Bolognia, “Aging skin," The American Journal of Medicine, vol. 98, no. 1, pp. S99-S103, 1995. 